\title{
Asymmetry Effects in Optical Properties of a Gold Nanotube
}

Afshin Moradi ( $\nabla$ a.moradi@kut.ac.ir )

Kermanshah University of Technology https://orcid.org/0000-0003-1055-0893

\section{Research Article}

Keywords: Symmetry-broken nanotube, Quasi-static approximation, Dispersion relation

Posted Date: March 9th, 2021

DOI: https://doi.org/10.21203/rs.3.rs-209681/v1

License: (c) (i) This work is licensed under a Creative Commons Attribution 4.0 International License. Read Full License 


\title{
Asymmetry effects in optical properties of a gold nanotube
}

\author{
Afshin Moradi* \\ Department of Engineering Physics, Kermanshah University of Technology, Kermanshah, Iran \\ a.moradi@kut.ac.ir
}

February 5, 2021

\begin{abstract}
Asymmetry effects in optical properties of an infinitely long gold nanotube is studied within the framework of the quasi-static approximation. In this way, incident, scattered and transmitted fields are represented with the appropriate electrostatic potentials in the cylindrical coordinates. The problem is investigated using a boundary-value approach. The expressions for the polarizability and dispersion of surface plasmons of the system are derived and numerical results, in the wavelength region $500 \mathrm{~nm}$ to $1000 \mathrm{~nm}$, show that instead of the one well-known resonance peak of the nanotube, one pair of resonance peaks appear, as a result of the coupling between the dipole and quadrupole modes. Then, scattering, absorption and extinction widths of the system are formulated. Also, the effective dielectric function of a composite of aligned symmetry-broken gold nanotubes is presented.
\end{abstract}

Keywords: Symmetry-broken nanotube; Quasi-static approximation; Dispersion relation

The problem of light interaction with a circular cylinder of infinite length was first studied by Ignatowski [1] in 1905 and then by Seitz [2] in 1906. This problem is a canonical boundary-value problem that can be solved with the separation of variables technique. In this way, in 1918 Lord Rayleigh [3] solved the boundary-value problem of scattering of a plane wave at normal incidence by a homogeneous dielectric cylinder. Also, Wait in 1955 [4], presented a solution for the problem of a plane wave incident obliquely on a circular cylinder of infinite length. The formal solution of the problem of the scattering of a plane electromagnetic wave by a system of two coaxial, dielectric cylinders was suggested by Thilo [5] in a study of radiation pressure. Then, Adey [6] presented calculation and experimental results for the case of a metal cylinder surrounded by a dielectric layer. Furthermore, Roumeliotis et al. $[7,8]$ studied the scattering of an electromagnetic plane wave by an infinite metallic cylinder eccentrically coated by another dielectric one.

Let us note that in all of the above mentioned works, because the overall size of a circular cylinder (or a circular tube) is large with respect to the wavelength of the excitation field, the absorption spectrum is weak compared with the scattering spectrum. Therefore, the scattering behaviors of such systems have been investigated, only. However, in the nano-world problems, we may deal with electromagnetic interaction with a nanowire or a nanotube, where the overall sizes of these obstacles are small. Therefore, the optical extinction should change from primarily scattering to primarily absorbing and the dipole modes dominate the extinction spectra $[9,10,11,12]$. Note that the mentioned new problem may be analyzed within the quasi-static approximation (QSA) [13, 14], where incident, scattered and transmitted waves may be represented with the electrostatic potential. Using the QSA method, the quasi-electrostatic interaction with

${ }^{*}$ Tel.: (+98)9183312692 


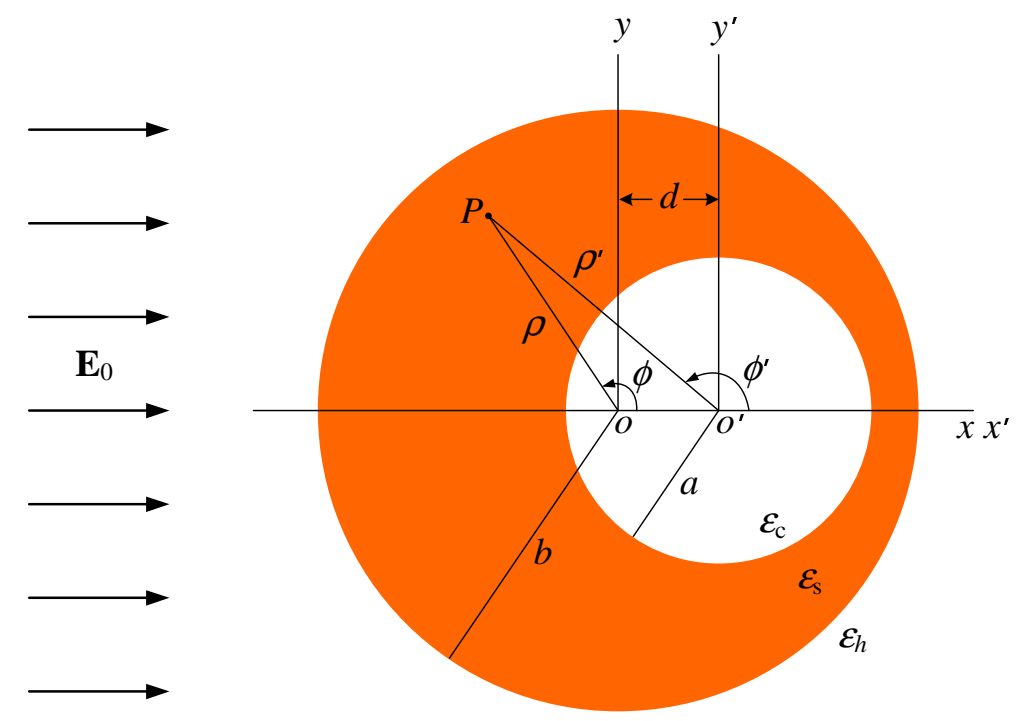

Figure 1: Cross section of a symmetry-broken gold nanotube placed into an electrostatic field. The shell and core surfaces have radii $a$ and $b$, respectively. the axis-to-axis separation begin $d$, where $d<b$.

a nanowire and a nanotube is studied in [12]. Also, plasmon hybridization in symmetry-broken metallic nanotubes are discussed by us in Ref. [15]. Furthermore, Ekeroth and Lester [16, 17] studied the optical characteristics of metallic nanotubes with broken symmetry using the integral method.

Recently, Starkov et al. [18] studied the optical properties of two eccentric cylinders in a uniform electric field. They described the fields in different regions by potentials which are solutions of the Laplace equation in the bipolar coordinate system. This involves a mathematical difficulty, as the particular solutions of the Laplace equation which have the correct behavior at infinity display a singularity in a point at finite distance which is inherent in the coordinate system used. However, they investigated the electric field distribution and polarizability of the structure. Also, the effective dielectric function of a periodic set of eccentric cylinders is derived.

As far as can be ascertained by this author, the problem of quasi-electrostatic interaction with a symmetry-broken metallic nanotube has not been published in the cylindrical coordinates. It is the purpose of this paper to give a solution for this problem that generates multipoles of all orders.

Fig. 1 shows cross section of a cylindrical metallic nanoshell surrounding a cylindrical nanocore displaced from the nanoshell axis by $d$, where $a$ and $b(b>a)$ are the nanocore and nanoshell radii, respectively. The relative dielectric constants of the host medium, the shell, and the cylindrical core are denoted by $\varepsilon_{h}, \varepsilon_{s}$, and $\varepsilon_{c}$, respectively. In the numerical result, we will consider a symmetry-broken metallic nanotube with a gold shell and a silica core. In the local response approximation, a Drude-Lorentz dielectric function for gold, which agrees with the experimental data in the wavelength region $500 \mathrm{~nm}$ to $1000 \mathrm{~nm}$, has been proposed as follows $[19,20,21]$

$$
\varepsilon_{s}(\omega)=\varepsilon_{\infty}-\frac{\omega_{f}^{2}}{\omega\left(\omega+i \gamma_{f}\right)}-\frac{\chi \omega_{b}^{2}}{\omega\left(\omega+i \gamma_{b}\right)-\omega_{b}^{2}},
$$

with model parameters $\varepsilon_{\infty}=5.9673, \omega_{f}=8.7411 \mathrm{eV}, \gamma_{f}=0.658 \mathrm{eV}, \omega_{b}=2.6885 \mathrm{eV}, \gamma_{f}=0.4337 \mathrm{eV}$ 
and $\chi=1.09$. Note that $\varepsilon_{\infty}$ is the high-frequency dielectric constant of gold, which accounts for the polarization of the positive ion core; $\omega_{f}$ and $\omega_{b}$ are the plasma frequency and resonance frequency of the free and bound electrons, respectively; $\gamma_{f}$ and $\gamma_{b}$ are the damping rates of the free and bound electrons, respectively; $\omega$ is the angular frequency of the incident electromagnetic wave and $\chi$ is the oscillator strength. In addition, we will use $\varepsilon_{c}=2.13$ [22] as the dielectric constant of the silica core.

Now, assume the system is exposed by an incoming weak plane wave characterized by its electric field strength $\mathbf{E}$ in the cross-section of the system and magnetic induction $\mathbf{B}$ parallel to the nanotube axis. We will use the QSA, i.e., take curl $\mathbf{E}=0$. The problem is then formally equivalent to an electrostatic problem with $E=-\nabla \Phi$ that obeys Laplace's equation [23, 24] and the electric field derives from a potential function $\Phi$. In this case, as shown in Fig. 1, the incident electric field is considered as $\mathbf{E}=E_{0} \exp (-i \omega t) \mathbf{e}_{x}$, where $E_{0}$ stands for the amplitude of this incident field. Also, $\mathbf{e}_{x}$ being the unit vector along the $x$-axis. We will therefore consider an exciting $\mathbf{E}$ field in the whole space; because of the QSA it represents quite satisfactorily the incoming $\mathbf{E}$ field of the plane wave in the neighbourhood of the system where the boundary conditions have to be stated.

The applied electric potential with respect to the xoy reference frame can be written as

$$
\Phi_{\mathrm{app}}=-E_{0} x=-E_{0} \rho \cos \phi,
$$

where the time dependence $\exp (-i \omega t)$ tacitly assumed. In the presence of the symmetry-broken nanotube the scattered electric field potential with respect to the xoy reference frame can be written as

$$
\Phi_{\text {sca }}=\sum_{n=1}^{\infty} A_{n}\left(\frac{b}{\rho}\right)^{n} \cos n \phi, \quad \rho \geq b .
$$

The transmitted electric field potential inside the shell with respect to the $x^{\prime} o^{\prime} y^{\prime}$ reference frame can be expressed, as

$$
\Phi_{s}=\sum_{n=1}^{\infty}\left[B_{n}\left(\frac{a}{\rho^{\prime}}\right)^{n}+C_{n}\left(\frac{\rho^{\prime}}{a}\right)^{n}\right] \cos n \phi^{\prime},
$$

while the electric field potential inside the core is then given by

$$
\Phi_{c}=\sum_{n=1}^{\infty} D_{n}\left(\frac{\rho^{\prime}}{a}\right)^{n} \cos n \phi^{\prime},
$$

where the factors of $a$ and $b$ are inserted so that the coefficients $A_{n}, B_{n}, C_{n}$ and $D_{n}$ have identical units. The boundary conditions on the shell and core surfaces are

$$
\begin{gathered}
\left.\Phi_{c}\right|_{\rho^{\prime}=a}=\left.\Phi_{s}\right|_{\rho^{\prime}=a^{\prime}}, \\
\left.\varepsilon_{\mathcal{c}} \frac{\partial \Phi_{c}}{\partial \rho^{\prime}}\right|_{\rho^{\prime}=a}=\left.\varepsilon_{s} \frac{\partial \Phi_{\mathrm{s}}}{\partial \rho^{\prime}}\right|_{\rho^{\prime}=a}, \\
\left.\Phi_{s}\right|_{\rho=b}=\left.\Phi_{\mathrm{app}}\right|_{\rho=b}+\left.\Phi_{\mathrm{sca}}\right|_{\rho=b}, \\
\left.\varepsilon_{s} \frac{\partial \Phi_{s}}{\partial \rho}\right|_{\rho=b}=\left.\varepsilon_{h} \frac{\partial\left[\Phi_{\mathrm{app}}+\Phi_{\mathrm{sca}}\right]}{\partial \rho}\right|_{\rho=b} .
\end{gathered}
$$

Substituting the potentials gives

$$
\sum_{n=1}^{\infty} D_{n} \cos n \phi^{\prime}=\sum_{n=1}^{\infty}\left[B_{n}+C_{n}\right] \cos n \phi^{\prime},
$$




$$
\begin{gathered}
\mathcal{E}_{c} \sum_{n=1}^{\infty} n D_{n} \cos n \phi^{\prime}=\varepsilon_{s} \sum_{n=1}^{\infty} n\left[-B_{n}+C_{n}\right] \cos n \phi^{\prime}, \\
\left.\sum_{n=1}^{\infty}\left[B_{n}\left(\frac{a}{\rho^{\prime}}\right)^{n}+C_{n}\left(\frac{\rho^{\prime}}{a}\right)^{n}\right] \cos n \phi^{\prime}\right|_{\rho=b}=-E_{0} b \cos \phi+\sum_{n=1}^{\infty} A_{n} \cos n \phi, \\
\left.\varepsilon_{s} b \sum_{n=1}^{\infty} \frac{\partial}{\partial \rho}\left[B_{n}\left(\frac{a}{\rho^{\prime}}\right)^{n}+C_{n}\left(\frac{\rho^{\prime}}{a}\right)^{n}\right] \cos n \phi^{\prime}\right|_{\rho=b}=-\varepsilon_{h} E_{0} b \cos \phi-\varepsilon_{h} \sum_{n=1}^{\infty} n A_{n} \cos n \phi .
\end{gathered}
$$

We next multiply the first two equations by $\cos m \phi^{\prime}$ and the second two equations by $\cos m \phi$ and integrate, respectively, with respect to $\phi^{\prime}$ and $\phi$ between 0 and $2 \pi$. Using the orthogonality relation

$$
\int_{0}^{2 \pi} \cos m \phi \cos n \phi d \phi=\pi \delta_{m n}
$$

where $\delta_{m n}$ is the Kronecker delta, we obtain

$$
\begin{gathered}
D_{m}=B_{m}+C_{m}, \\
\varepsilon_{c} D_{m}=\varepsilon_{s}\left[-B_{m}+C_{m}\right], \\
\sum_{n=1}^{\infty} S_{m n} B_{n}+\sum_{m=1}^{\infty} T_{m n} C_{n}=-E_{0} b \delta_{1 m}+A_{m}, \\
\varepsilon_{s} \sum_{n=1}^{\infty} U_{m n} B_{n}+\varepsilon_{s} \sum_{n=1}^{\infty} V_{m n} C_{n}=-\varepsilon_{h} E_{0} b \delta_{1 m}-m \varepsilon_{h} A_{m} .
\end{gathered}
$$

where the matrix elements are given by

$$
\begin{gathered}
S_{m n}=\left.\frac{a^{n}}{\pi} \int_{0}^{2 \pi} \frac{\cos n \phi^{\prime}}{\rho^{\prime n}}\right|_{\rho=b} \cos m \phi d \phi, \\
T_{m n}=\left.\frac{a^{-n}}{\pi} \int_{0}^{2 \pi} \rho^{\prime n} \cos n \phi^{\prime}\right|_{\rho=b} \cos m \phi d \phi, \\
U_{m n}=\frac{b a^{n}}{\pi} \int_{0}^{2 \pi} \frac{\partial}{\partial \rho}\left[\frac{\cos n \phi^{\prime}}{\rho^{\prime n}}\right]_{\rho=b} \cos m \phi d \phi, \\
V_{m n}=\frac{b a^{-n}}{\pi} \int_{0}^{2 \pi} \frac{\partial}{\partial \rho}\left[\rho^{\prime n} \cos n \phi^{\prime}\right]_{\rho=b} \cos m \phi d \phi .
\end{gathered}
$$

In order to evaluate these matrix elements, we use the addition theorem for cylindrical Bessel and Neumann functions in the limiting forms for small arguments, as $[7,8]$

$$
\begin{aligned}
\rho^{\prime n} \cos n \phi^{\prime} & =\sum_{k=1}^{\infty}(-1)^{n-k} \frac{n !}{k !(n-k) !} d^{n-k} \rho^{k} \cos k \phi, \\
\frac{\cos n \phi^{\prime}}{\rho^{\prime n}} & =\sum_{k=1}^{\infty} \frac{(k-1) !}{(n-1) !(k-n) !} d^{k-n} \frac{\cos k \phi}{\rho^{k}} .
\end{aligned}
$$

We then obtain

$$
S_{m n}=\frac{a^{n}}{b^{m}} \frac{(m-1) !}{(n-1) !(m-n) !} d^{m-n}
$$




$$
\begin{aligned}
T_{m n} & =(-1)^{n-m} \frac{b^{m}}{a^{n}} \frac{n !}{m !(n-m) !} d^{n-m}, \\
U_{m n} & =-m \frac{a^{n}}{b^{m}} \frac{(m-1) !}{(n-1) !(m-n) !} d^{m-n}, \\
V_{m n} & =m(-1)^{n-m} \frac{b^{m}}{a^{n}} \frac{n !}{m !(n-m) !} d^{n-m} .
\end{aligned}
$$

where $S_{m n}=0$ for $m<n$ and $T_{m n}=0$ for $m>n$. Also, note that $U_{m n}=-m S_{m n}$ and $V_{m n}=m T_{m n}$.

Now, we study the static dipole polarizability of the system. To obtain the static dipole polarizability, we express $A_{1}$ in terms of $E_{0}$. To do this, we obtain $A_{1}$ by using the resulting equations based on the two lowest-order modes, $n=1$ and $n=2$, in Eqs. (10)-(13) [after changing $m$ to $n$ and $n$ to $m]$. We find

$$
A_{1}=\frac{\varepsilon_{S}+\varepsilon_{h}}{\varepsilon_{S}-\varepsilon_{h}} \frac{\left(S_{11}+\Psi T_{11}\right)\left(S_{22}+Y T_{22}\right)-\Psi T_{12} S_{21}}{\left(S_{11}+Y T_{11}\right)\left(S_{22}+Y T_{22}\right)-Y T_{12} S_{21}} E_{0} b,
$$

where

$$
\begin{aligned}
& Y=\frac{\left(\varepsilon_{h}+\varepsilon_{s}\right)\left(\varepsilon_{s}+\varepsilon_{\mathcal{c}}\right)}{\left(\varepsilon_{h}-\varepsilon_{s}\right)\left(\varepsilon_{s}-\varepsilon_{c}\right)}, \\
& \Psi=\frac{\left(\varepsilon_{h}-\varepsilon_{s}\right)\left(\varepsilon_{s}+\varepsilon_{c}\right)}{\left(\varepsilon_{h}+\varepsilon_{s}\right)\left(\varepsilon_{s}-\varepsilon_{c}\right)},
\end{aligned}
$$

and

$$
\begin{gathered}
S_{11}=\frac{a}{b}, \quad S_{22}=\frac{a^{2}}{b^{2}}, \quad T_{11}=\frac{b}{a}, \quad T_{22}=\frac{b^{2}}{a^{2}}, \\
S_{21}=\frac{a}{b^{2}} d, \quad T_{12}=-2 \frac{b}{a^{2}} d .
\end{gathered}
$$

Note that $S_{11}\left(S_{22}\right)$ and $T_{11}\left(T_{22}\right)$ are coupling constants of wire and channel dipole (quadrupole) surface plasmons, respectively, and $T_{12}$ and $S_{21}$ are the dipole-quadrupole and quadrupole-dipole coupling constants of channel and wire surface plasmons, respectively [20, 21].

At this stage, the static dipole polarizability of a symmetry-broken nanotube in a medium with dielectric constant $\varepsilon_{h}$ may be written as [12]

$$
\alpha=2 \frac{A_{1}}{E_{0} b} .
$$

Eq. (25) is the (complex) normalized transversal polarizability of a symmetry-broken nanotube of sub-wavelength diameter in the QSA. It is clear that Eq. (25) is equal with the normalized transversal polarizability of a regular nanotube when $d \rightarrow 0$ [12]. In this case we have

$$
\alpha_{(d=0)}=2 \frac{\varepsilon_{s}+\varepsilon_{h}}{\varepsilon_{S}-\varepsilon_{h}} \frac{S_{11}+\Psi T_{11}}{S_{11}+Y T_{11}}
$$

Fig. 2 shows variations of the absolute value of $\alpha$ with respect to the wavelength for a symmetrybroken gold nanotube with dimensions $a=15 \mathrm{~nm}$ and $b=20 \mathrm{~nm}$ for five different values of coreoffsets as $d=0,1,2,3$ and $4 \mathrm{~nm}$. As can be seen in this figure by increasing core-offset, the dipole surface plasmon resonance of the system undergoes a red shift. Also, at large core-offsets due to an increase in the dipole-quadrupole surface plasmon interaction strength, a noticeable quadrupole surface plasmon resonance appears. 


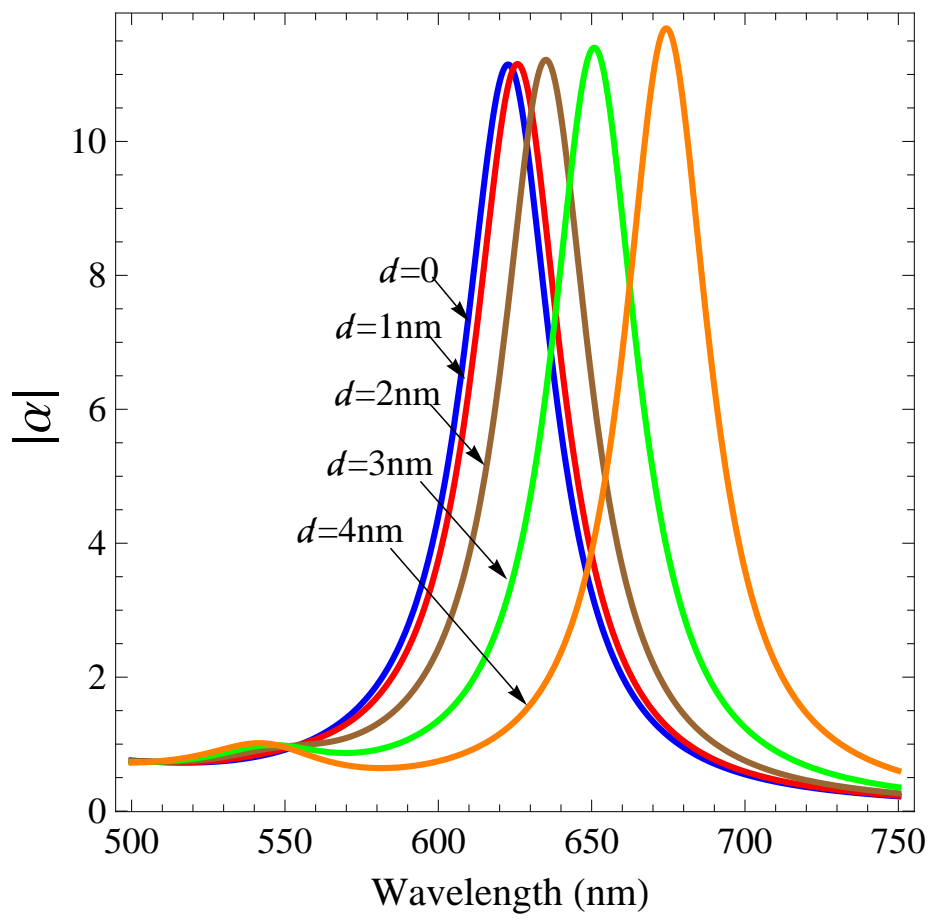

Figure 2: Variations of the absolute value of $\alpha$ with respect to the wavelength for a symmetry-broken gold nanotube with dimensions $a=15 \mathrm{~nm}$ and $b=20 \mathrm{~nm}$ and the following core-offsets: $d=0,1,2,3$ and $4 \mathrm{~nm}$. Here, we have used Eq. (1) as Drude-Lorentz dielectric function of gold nanotube. Also, we have used $\varepsilon_{m}=1$ and $\varepsilon_{\mathcal{c}}=2.13$.

If the denominator of Eq. (24) becomes minimum, then transversal polarizability of the system experiences a resonant enhancement which for the case of small damping simplifies to

$$
\left(S_{11}+Y T_{11}\right)\left(S_{22}+Y T_{22}\right)-Y T_{12} S_{21}=0 .
$$

Eq. (27) is the dispersion relation of surface plasmons of a symmetry-broken gold nanotube, when coupling occurs between the dipole and quadrupole modes and any damping constant is zero. For the benefit of the reader, Eq. (27) is derived directly from an alternative approach in the Appendix A.

Also, the scattering, absorption and extinction widths of the system in the QSA can be calculated using [12]

$$
\begin{gathered}
Q_{\text {sca }}=\frac{\pi^{2}}{16} \varepsilon_{h}^{3 / 2}\left(\frac{b \omega}{c}\right)^{3}|\alpha|^{2}, \\
Q_{\mathrm{abs}}=-\frac{\pi}{2} \varepsilon_{h}^{1 / 2}\left(\frac{b \omega}{c}\right)\left[\operatorname{Re}[i \alpha]+\frac{\pi}{8} \varepsilon_{h}\left(\frac{b \omega}{c}\right)^{2}|\alpha|^{2}\right], \\
Q_{\mathrm{ext}}=-\frac{\pi}{2} \varepsilon_{h}^{1 / 2} \frac{b \omega}{c} \operatorname{Re}[i \alpha],
\end{gathered}
$$

respectively, in units of the geometric width $2 b$. Note that Re denotes the real part and $c$ is the light speed in free space. 
Finally, the effective dielectric function $\varepsilon_{\text {eff }}$ of a composite medium of aligned symmetrybroken metallic nanotubes embedded in a host medium with relative dielectric constant of $\varepsilon_{h}$ may be presented in the QSA, as [12]

$$
\frac{\varepsilon_{\text {eff }}-\varepsilon_{h}}{\varepsilon_{\text {eff }}+\varepsilon_{h}}=\frac{1}{2} f \alpha,
$$

where $f$ is the volume fraction of the embedded nanotubess and $\alpha$ is the dipole polarizability of a nanotubes, as can be seen in Eq. (25). Substituting Eq. (25) into Eq. (31), leads to

$$
\frac{\varepsilon_{\text {eff }}-\varepsilon_{h}}{\varepsilon_{\text {eff }}+\varepsilon_{h}}=f \frac{\varepsilon_{s}+\varepsilon_{h}}{\varepsilon_{s}-\varepsilon_{h}} \frac{\left(S_{11}+\Psi T_{11}\right)\left(S_{22}+Y T_{22}\right)-\Psi T_{12} S_{21}}{\left(S_{11}+Y T_{11}\right)\left(S_{22}+Y T_{22}\right)-Y T_{12} S_{21}} .
$$

Eq. (32) is the effective permittivity formula of a composite of aligned symmetry-broken gold nanotubes, in the QSA.

In conclusion, we have shown that the quasi-electrostatic field interaction with a symmetrybroken gold nanotube generates multipoles of all orders. By considering the two lowest order terms in the multipole expansion coefficients, we have observed that coupling occurs between the dipole and quadrupole modes of the system. We have shown that the increasing of the core-offset, red-shifts the dipole plasmon resonance, where at large core-offsets, a noticeable quadrupole surface plasmon resonance appears. Furthermore, within the framework of the QSA, the formulas of scattering, absorption and extinction widths of a symmetry-broken gold nanotube have been presented. Finally, the effective dielectric function of a composite of aligned symmetrybroken gold nanotubes has been derived.

\section{APPENDIX: A DIRECT DERIVATION OF DISPERSION RELATION OF SURFACE PLASMONS}

The dispersion relation of surface plasmons of a symmetry-broken gold nanotube, when coupling occurs between the dipole and quadrupole modes, can be obtained by using the general results presented in Ref. [15]. To do this, from [15], we have

$$
\begin{aligned}
& \mathbf{B}=\mathrm{MC}, \\
& \mathbf{C}=\mathbf{N B} .
\end{aligned}
$$

In these equations, the components of vectors $\mathbf{B}$ and $\mathbf{C}$ are $B_{m}$ and $C_{m}$, respectively and the matrices $\mathbf{M}$ and $\mathbf{N}$ have the elements

$$
\begin{aligned}
M_{m n} & =\left(\varepsilon_{s}-\varepsilon_{h}\right) \frac{I_{m-n}(q d) I_{m}(q b) K_{m}(q b) K_{m}^{\prime}(q b)}{K_{n}(q a)\left[\varepsilon_{h} I_{m}(q b) K_{m}^{\prime}(q b)-\varepsilon_{s} I_{m}^{\prime}(q b) K_{m}(q b)\right]}, \\
N_{m n} & =\left(\varepsilon_{c}-\varepsilon_{s}\right) \frac{I_{n-m}(q d) I_{m}(q a) K_{m}(q a) I_{m}^{\prime}(q a)}{I_{n}(q b)\left[\varepsilon_{s} I_{m}(q a) K_{m}^{\prime}(q a)-\varepsilon_{c} I_{m}^{\prime}(q a) K_{m}(q a)\right]} .
\end{aligned}
$$

Note that in Eqs. (A-3) and (A-4), $q$ is the wavenumber of the surface plasmons [15], $I_{m}^{\prime}(x)=$ $d I_{m}(x) / d x$ and $K_{m}^{\prime}(x)=d K_{m}(x) / d x$. Now, from Eq. (A-1) and (A-2), we find

$$
(\mathbf{M N}-1) \mathbf{B}=0 .
$$

For general values of $m, n$ and $q$, the zeros of the determinant of the matrix ( $\mathbf{M N}-1)$ can be solved numerically by truncating the summations in order to obtain the dispersion relations of 
surface plasmons of the system. However, when $q a, q b$ and $q d$ are small, an analytical procedure can be developed. As $x \rightarrow 0$, we use the following limiting values [12]

$$
\begin{gathered}
I_{m}(x)=\frac{1}{m !}\left(\frac{x}{2}\right)^{m},(m \geq 0), \\
I_{m}^{\prime}(x)=\frac{1}{2(m-1) !}\left(\frac{x}{2}\right)^{m-1},(m \geq 1), \\
K_{m}(x)=\frac{(m-1) !}{2}\left(\frac{2}{x}\right)^{m},(m \geq 1), \\
K_{m}^{\prime}(x)=-\frac{m !}{4}\left(\frac{2}{x}\right)^{m+1},(m \geq 1) .
\end{gathered}
$$

Substitution of these limiting values in Eqs. (A-3) and (A-4), one obtains

$$
\begin{gathered}
M_{m n}=-\frac{(m-1) !}{(n-1) !(m-n) !}\left(\frac{a}{d}\right)^{n}\left(\frac{d}{b}\right)^{m} \frac{\varepsilon_{h}-\varepsilon_{s}}{\varepsilon_{h}+\varepsilon_{s}}, \quad(m \geq n), \\
N_{m n}=\frac{n !}{m !(n-m) !}\left(\frac{a}{d}\right)^{m}\left(\frac{d}{b}\right)^{n} \frac{\varepsilon_{s}-\varepsilon_{c}}{\varepsilon_{s}+\varepsilon_{c}}, \quad(n \geq m) .
\end{gathered}
$$

Therefore, from Eqs. (A-1) and (A-2) for $m, n=1,2$, we find

$$
\begin{gathered}
B_{1}=-\frac{a}{b} \frac{\varepsilon_{h}-\varepsilon_{s}}{\varepsilon_{h}+\varepsilon_{s}} C_{1}, \\
B_{2}=-\frac{a d}{b^{2}} \frac{\varepsilon_{h}-\varepsilon_{s}}{\varepsilon_{h}+\varepsilon_{s}} C_{1}-\left(\frac{a}{b}\right)^{2} \frac{\varepsilon_{h}-\varepsilon_{s}}{\varepsilon_{h}+\varepsilon_{s}} C_{2}, \\
C_{1}=\frac{a}{b} \frac{\varepsilon_{s}-\varepsilon_{c}}{\varepsilon_{s}+\varepsilon_{c}} B_{1}-2 \frac{a d}{b^{2}} \frac{\varepsilon_{s}-\varepsilon_{c}}{\varepsilon_{s}+\varepsilon_{c}} B_{2}, \\
C_{2}=\left(\frac{a}{b}\right)^{2} \frac{\varepsilon_{s}-\varepsilon_{c}}{\varepsilon_{s}+\varepsilon_{c}} B_{2} .
\end{gathered}
$$

Finally, from Eqs. (A-8)-(A-11), we get Eq. (27).

\section{FUNDING}

No funding was received for this work.

\section{CONFLICTS OF INTEREST/COMPETING INTERESTS}

The author declares no conflicts of interest/competing interests.

\section{Availability OF DATA AND MATERIAL}

The data that supports the findings of this study are available within the article.

\section{Code availability}

Not applicable 


\section{Authors' CONTRIBUtions}

This article has been authored by a single author.

\section{REFERENCES}

[1] W. von Ignatowski, Reflexion elektromagnetischer Wellen an einem dünnen drähte, Ann. Phys. 18, 495-522 (1905).

[2] W. Seitz, Die beugung des lichtes an einem dünnen, zylindrischen drähte, Ann. Phys. 21, 1013-1029 (1906).

[3] L. Rayleigh, The dispersal of light by a dielectric cylinder, Philos. Mag. 36, 365-376 (1918).

[4] J. R. Wait, Scattering of a plane wave from a circular dielectric cylinder at oblique incidence, Can. J. Phys. 33, 189-195 (1955).

[5] G. Thilo, Der Strahlungsdruck auf Kreiszylinder aus beliebigem Material, Ann. Physik 367, 531-568 (1920).

[6] A. W. Adey, Scattering of electromagnetic waves by coaxial cylinders, Can. J. Phys. 34, 510520 (1956).

[7] J. A. Roumeliotis, J. G. Fikioris, and G. P. Gounaris, Electromagnetic scattering from an eccentrically coated infinite metallic cylinder, J. Appl. Phys. 51, 4488-4493 (1980).

[8] J. A. Roumeliotis and N. B. Kakogiannos, Scattering from an infinite cylinder of small radius embedded into a dielectric one, IEEE Trans. Microwave Theory Tech. 42, 463-470 (1994).

[9] L. Novotny, B. Hecht, Principles of Nano-Optics, Cambridge University Press, Cambridge, (2006).

[10] D. $\mathrm{Wu}, \mathrm{X}$. Liu, and B. Li, Localized surface plasmon resonance properties of two-layered gold nanowire: effects of geometry, incidence angle, and polarization, J. Appl. Phys. 109, 083540 (2011).

[11] R. M. A. Ekeroth, and M. Lester, Optical forces on silver homogeneous nanotubes: study of shell plasmonic interaction, Plasmonics 10, 989-998 (2015).

[12] A. Moradi, Canonical problems in the theory of plasmonics, Springer Series in Optical Sciences, vol 230. Springer, Switzerland, Cham (2020).

[13] A. Moradi, Surface and bulk plasmons in cylindrical electric-gyrotropic wires, J. Opt. Soc. Am. B 37, 2947-2955 (2020).

[14] A. Moradi, Optical properties of two-walled carbon nanotubes: quasi-static approximation, Eur. Phys. J. Plus 135, 611 (2020).

[15] A. Moradi, Plasmon hybridization in metallic nanotubes with a nonconcentric core, Opt. Commun. 282, 3368-3370 (2009).

[16] R. M. A. Ekeroth, and M. Lester, Optical properties of metallic nanotubes with broken symmetry: detection of eccentricity, Plasmonics 7, 579-587 (2012). 
[17] R. M. A. Ekeroth, and M. Lester, Detection of eccentricity in silver nanotubes by means of induced optical forces and torques, J. Opt. 17, 105002 (2015).

[18] A. S. Starkov, K. V. Korzenkov, and K. A. Starkov, Two eccentric cylinders in a uniform electric field, Nanosystems: Phys. Chem. Math. 8, 277-281 (2017).

[19] A. Vial, A.-S. Grimault, D. Macías, D. Barchiesi, and M. L. Chapelle, Improved analytical fit of gold dispersion: Application to the modeling of extinction spectra with a finite-difference time-domain method, Phys. Rev. 71, 085416 (2005).

[20] L. C. Ugwuoke, T. Mancal, and T. P. J. Kruger, Optical properties of a nanoegg-nanorod heterodimer: a quasi-static analysis, J. Opt. Soc. Am. B 37, A293-A303 (2020).

[21] L. C. Ugwuoke, T. Mancal, and T. P. J. Kruger, Plasmonic quantum yield enhancement of a single molecule near a nanoegg, J. Appl. Phys. 127, 203103 (2020).

[22] H. Wang, Y. Wu, B. Lassiter, C. L. Nehl, J. H. Hafner, P. Nordlander, and N. J. Halas, Symmetry breaking in individual plasmonic nanoparticles, Proc. Natl. Acad. Sci. USA 103, 1085610860 (2006).

[23] F. Mangini, N. Tedeschi, F. Frezza, and A. Sihvola, Electromagnetic interaction with two eccentric spheres, J. Opt. Soc. Am. A 31, 783-789 (2014).

[24] S. J. Norton and T. Vo-Dinh, Optical Fano resonances in a nonconcentric nanoshell, Appl. Opt 55, 2611-2618 (2016). 


\section{Figures}

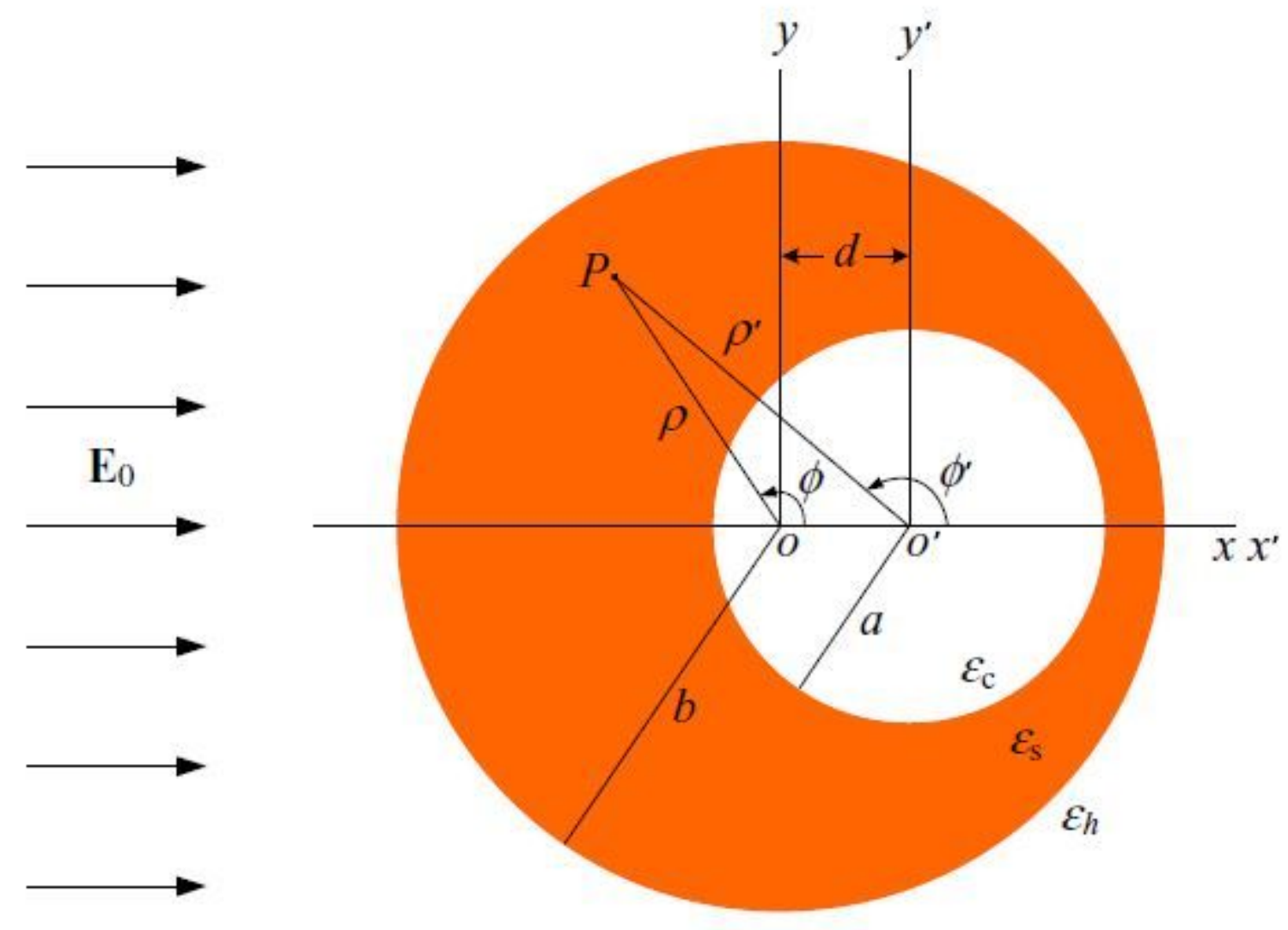

Figure 1

Cross section of a symmetry-broken gold nanotube placed into an electrostatic field. The shell and core surfaces have radii a and $b$, respectively. the axis-to-axis separation begin $d$, where $d<b$. 


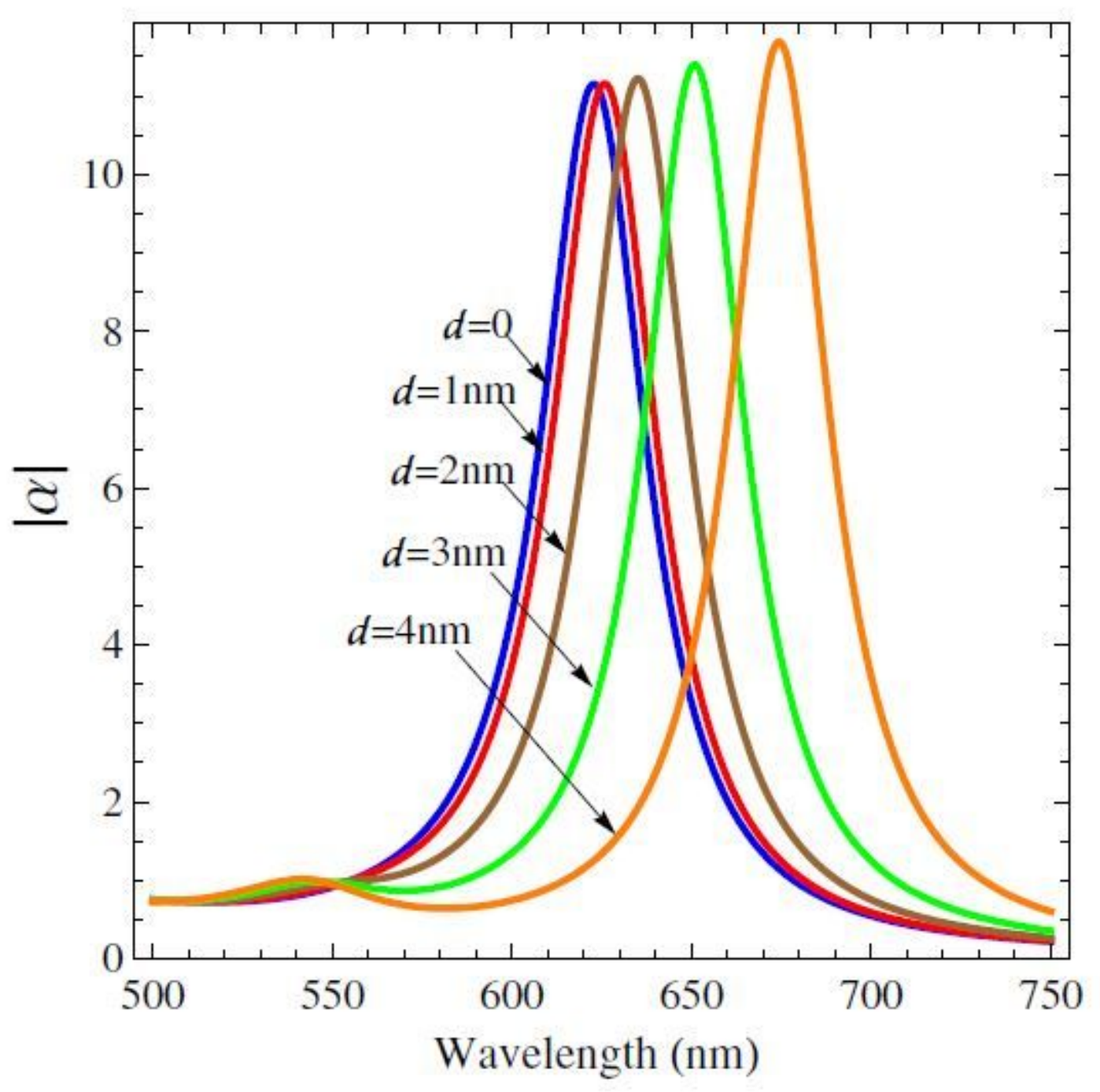

Figure 2

Variations of the absolute value of a with respect to the wavelength for a symmetry-broken gold nanotube with dimensions $a=15 \mathrm{~nm}$ and $b=20 \mathrm{~nm}$ and the following core-offsets: $d=0,1,2,3$ and $4 \mathrm{~nm}$. Here, we have used Eq. (1) as Drude-Lorentz dielectric function of gold nanotube. Also, we have used $\varepsilon \mathrm{m}$ $=1$ and $\varepsilon \mathrm{c}=2.13$. 